\title{
VIRTUAL SENSING TECHNIQUES FOR THE ESTIMATION OF JOINTS CONCEPT MODELS PARAMETERS
}

\author{
Simone Gallas*, Jan Croes, Stijn Jonckheere, Jelle Bosmans, Wim Desmet \\ ${ }^{1}$ KU Leuven \\ Department of Mechanical Engineering Celestijnenlaan 300, 3001 Leuven, Belgium \\ simone.gallas@kuleuven.be,jan.croes@kuleuven.be, stijn.jonckheere@kuleuven.be, \\ jelle.bosmans@kuleuven.be,wim.desmet@kuleuven.be \\ ${ }^{2}$ DMMS Core Lab, Flanders Make
}

\begin{abstract}
Within the current trend towards lightweight and multi-material structures, the design of structural joints has even more become a topic of particular interest. Including these joints in models that can be used in the design, monitoring and control of structures dynamics behavior is fundamental. In front of the multiple modelling strategies, the engineer always has compromise between accuracy and simplicity, according to the application requirements. On the one hand, high complexity models have the potential to be very accurate but they require long modelling and simulation times and an extensive set of to be identified parameters. On the other hand, simple concept models, that approximate the behavior on a system level, allows a still representative accuracy with a lower computational cost for a large set of applications. In this latter case, it is more challenging to identify the parameters and their corresponding application range. In this context the parameter estimation techniques that inherently account for the model inaccuracies gain importance. This work proposes a new methodology for the estimation of the parameters for an adhesive joint concept model. The novelty involves a virtual sensing strategy, where the model and the experiments are used in a coupled fashion, to obtain with a limited number of simple non-intrusive measurements, an extended set of virtual data. Particularly a stochastic estimator has been used: the Kalman Filter brings together measurements from accelerometers with a reduced order model. Sensitivity and observability analysis are performed to set the initial configuration of the algorithm. Finally the work shows, numerically and experimentally, that this methodology allows to estimate the parameters of an adhesive joint concept model, resulting in an accurate representation of the system.
\end{abstract}

Keywords: Joint modelling, parameter estimation, virtual sensing, Kalman filter, structural dynamics 


\section{INTRODUCTION}

In lightweight systems, where different types of material are connected together, the joining technologies have a dominant effect on the overall behavior and they must be properly considered in the modelling phase. Mechanical joints often have an important non-linear influence on the transient behavior of the system, due to local changes in stiffness and damping. Hence in that respect having a non-negligible influence on the lifetime evaluation of the whole structure. It is evident that the analysis and monitoring of these connections is fundamental [1].

From the system level point of view, a detailed modelling for each of the joints present in a certain assembly is a cumbersome process as it includes many local changes in the mesh geometry and hence a significantly large model in terms of degrees of freedom. Then, the modelling strategy selection for the joints becomes really important; engineers have to find an optimum between complex joints models, requiring an extensive modelling effort and long simulation times, or simple concept models where it is unclear which approach yields at accurate results. For this latter case, the model parameters are usually fewer but they are difficult to identify a priori. For these reasons their estimation is crucial and particularly challenging. Often the parameters are not practically accessible in-situ, example given for the elastic module of a glue, or a bolt stiffness; in other cases several physical characteristics are condensed in few model parameters, that are not directly linked to the reality and thus, they are not directly measurable from experiments.

This article proposes to identify the parameters of these concept joint models by upgrading them to the Digital Twin level [2]: the purely mathematical representation, the model, is kept connected to the reality in a closed-loop fashion through a number measurements, resulting in a more reliable and accurate analysis tool. The Digital Twin can provide full-field information of the system and a selection of this information, later called virtual sensors, can be used for an accurate estimation of the model parameters. One of the main advantages of these novel virtual sensing methodologies is the feasibility of in-situ estimation, based on few non-intrusive measurements. Furthermore it only requires time-domain signals, opening the possibility of estimation in operational conditions. Finally there is the potential for improved accuracy with respect to the traditional identification methods. Essential readings about the traditional identification methods for parametric models are the surveys by Mottershead [3] and Åström [4].

The presented work consists of the introduction and experimental validation of a new virtual sensing technique for parameter estimation. It is based on a stochastic Kalman filter estimator [5] that elaborates a reduced order model and few dynamical measurements, together with their respective uncertainties, the process and measurements noises. The Kalman filter is fed with 4 real sensors signals attached to the test-rig and it is used to estimate the corresponding 4 virtual sensors, in this case accelerometers. The algorithm is repeated for different values of the parameter to be estimated, and the optimal parameter value is the one that minimize a cost function related to the error between the real and the virtual sensors. The selected validation case consists in two plates connected with an adhesive lap joint, where the elastic module of one of the two plates is the parameter to be estimated. It is relevant to notice that with the proposed methodology, the parameter can be positively estimated in a non-intrusive way, without sensors or excitation systems attached to the component to whom the parameter physically belongs.

The next 5 sections introduce the 5 fundamental steps of the proposed methodology: Model definition, Sensitivity analysis, Sensors configuration and observability, Experimental data, and Estimation. They are presented in the chronological and logical order that should be followed for the methodology implementation. For this reason this work could be also seen as a practical 
guideline for this new parameter estimation technique.

\section{MODEL DEFINITION}

\subsection{Finite element modelling}

In the current engineering environment the most well-known modelling tool for structural analysis is the Finite Element (FE) method. For this reason the methodology reported in this article is based on a FE model, even though other modelling approaches could have been used with similar results. In a FE model the system is spatially discretized in a number of $n_{\text {elem }}$ elements, defined by $n_{\text {nodes }}$ nodes. Each node is represented by a number of degrees of freedom for a total of $n_{\text {dofs }}$ degrees of freedom in the model, and for each degree of freedom there is an equation describing its interactions with the other ones. Of course these interactions are defined by the physical laws, considering the applied external forces, the elements topology and the elements material properties, mainly stiffness and mass. The model is finally a mathematical representation of the real system, consisting in a system of $n_{\text {dofs }}$ equations to be solved. In this work the commercial FE framework SIMCENTER 3D - NX Nastran solver has been used. The system considered consists in two aluminum plates with dimensions $221 \mathrm{~mm}$ x $132 \mathrm{~mm}$ x 4mm, adhesively bonded together with commercial super-glue LOCTITE 41. The lap joint involves the whole width of the plates, and the overlap measures $20 \mathrm{~mm}$ in the length direction. Similar single lap joints are modelled also in the articles by He [6] and Van Belle [7]. In this work the system is clamped at one side, and the elastic module of the clamped plate is the parameter to be estimated.

The FE model includes 60260 hexagonal solid elements (8 nodes each), 73920 nodes and 3 translational degrees of freedom per node. At the adhesive location the substrate mesh is refined, resulting in cubic shaped elements with dimension $1 \mathrm{~mm}$. The thickness of the plates is subdivided into 4 elements, whereas the adhesive part consists in one only layer of solid elements with thickness $0.5 \mathrm{~mm}$. The material and geometric properties are set as shown in Table 1 and the meshed model can be seen in Figure 1. The coordinate system that will be used through all the article, is centered at the right corner opposite to the clamping, the $\mathrm{x}$ direction is along the width and the $y$ is along the length of the system.

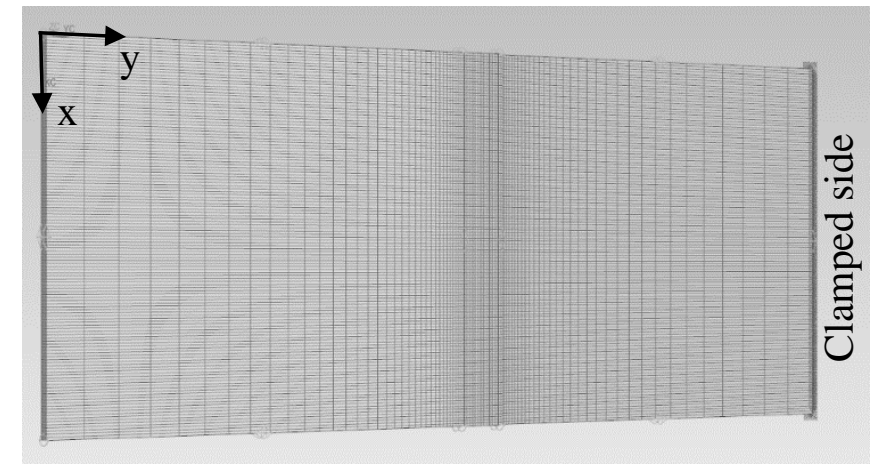

Figure 1: meshed Finite Element model

\begin{tabular}{lll}
\hline & Clamped Plate & Top Plate \\
\hline Length & $0.221 \mathrm{~m}$ & $0.221 \mathrm{~m}$ \\
\hline Width & $0.132 \mathrm{~m}$ & $0.132 \mathrm{~m}$ \\
\hline Thickness & $0.004 \mathrm{~m}$ & $0.004 \mathrm{~m}$ \\
\hline Density & $2692 \mathrm{~kg} \mathrm{~m}^{\wedge}-3$ & $2692 \mathrm{~kg} \mathrm{~m}^{\wedge}-3$ \\
\hline Elastic Module & $?$ & $69 \mathrm{GPa}$ \\
\hline
\end{tabular}

Table 1: System physical properties 


\subsection{Parametric model order reduction}

The model is not directly solved within SIMCENTER 3D environment, but its input file is exported into Matlab, where the assembled stiffness and mass matrixes are elaborated and the model is reduced, by solving the eigenvalues problem. However, in this mathematical operation the explicit influence of the parameter on the system equations would get lost and thus its estimation would not be possible. To overcome this issue, a parametric model order reduction (PMOR) scheme is adopted. Benner et al. [8] provide a wide survey on the different schemes available in literature. In this article, the used PMOR technique is similar to the one introduced by Naets [9], where it is used for a non-linear estimation within an Extended Kalman filter. It consists of the following steps: first, the parametric space has been sampled with a discrete number of values equally spaced around the expected one; second, the model has been locally reduced, meaning that the eigenvalues and the eigenvectors have been separately computed for each different parameter sample (Figure 2). The model can later be interpolated between those parametric samples, and then used in simulations or estimations.
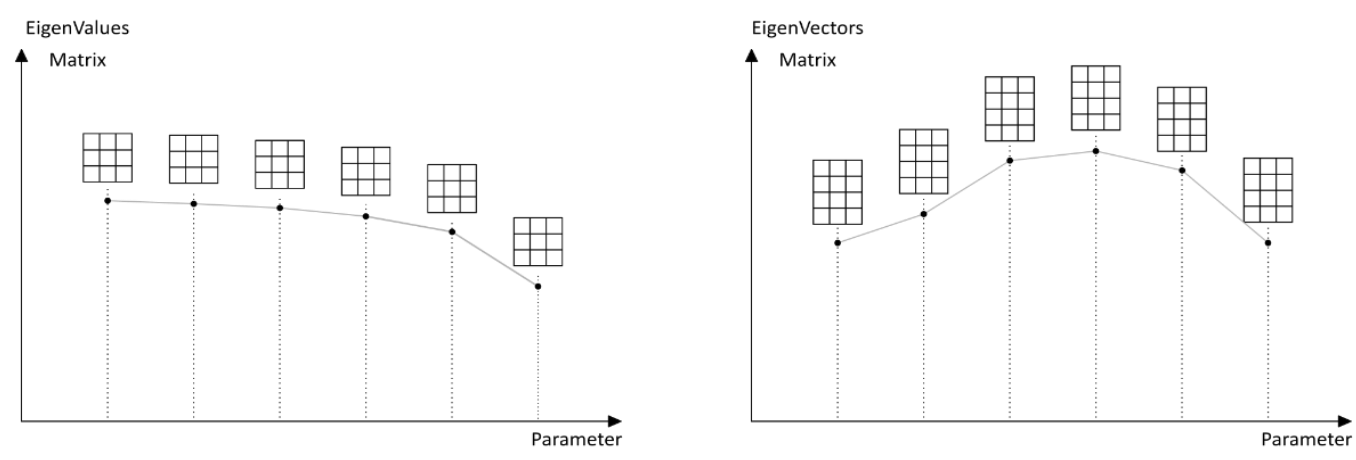

Figure 2: Parametric model order reduction scheme

\subsection{State-space formulation}

Given the equation of motion for the FE model:

$$
M \ddot{x}+C \dot{x}+K x=F
$$

Where $\mathrm{M}, \mathrm{C}$ and $\mathrm{K}$ are respectively the mass, damping and stiffness matrix, the vector $x$ contains the degrees of freedom and $\mathrm{F}$ is the input forces vector; the nodal coordinates can be projected on the modal space, and the original system of a number of $n_{\text {dofs }}$ equations is reduced to a system of $n_{\text {modes }}$ equations. This procedure has been repeated for several parameter values, as described in the previous section.

The reduced order model can then be written as:

$$
I \ddot{q}+2 \zeta \Lambda \dot{q}+\Lambda^{2} q=\Psi^{\mathrm{T}} F
$$

Where $I$ is the identity matrix, $\Lambda$ is the eigenvalues matrix, $\zeta$ is the modal damping factors matrix, $\Psi$ is the eigenvector matrix with dimensions $n_{\text {dofs }} \times n_{\text {modes }}$, and $F$ is again the input forces vector. The state space formulation allows at this point to pass from a second order ODE system to a first order ODE: 


$$
\left[\begin{array}{c}
\dot{q} \\
\ddot{q}
\end{array}\right]=\left[\begin{array}{cc}
0 & I \\
\Lambda^{2} & 2 \zeta \Lambda
\end{array}\right]\left[\begin{array}{c}
q \\
\dot{q}
\end{array}\right]+\left[\begin{array}{c}
0 \\
\Psi^{\mathrm{T}}
\end{array}\right][F]
$$

Let the state vector be $x=\left[\begin{array}{c}q \\ \dot{q}\end{array}\right]$, the system can then be rewritten in the condensed form:

$$
\begin{aligned}
& \dot{x}=A x+B u+w \\
& y=C x+D u+v
\end{aligned}
$$

The equations describe a continuous-time, linear, first order dynamical system where $x$ is the vector of the states (in this work, the modal participation factors), $\dot{x}$ is the derivative of the state vector, $u$ is the input vector (in this work a concentrated force), $A$ is the states matrix with dimensions $2 n_{\text {modes }} \times 2 n_{\text {modes }}, B$ is the input matrix with dimensions $2 n_{\text {modes }} \times 1$, and $w$ represents the process noise. The output equation (5) includes $y$ that is the output vector (in this exercise, the accelerometers signals), $C$ is the state-output matrix with dimensions $n_{\text {meas }} \times$ $2 n_{\text {modes }}, D$ is the input-output matrix (zero in this case) and finally $v$ is the measurement noise.

\section{SENSITIVITY ANALYSIS}

Having already computed the eigenvalues and eigenvectors of the problem for different values of the parameter to be estimated, a dynamical sensitivity analysis can be made. The purpose of this phase is to assess the influence of the parameter on the system dynamics, which is quantified by looking at how much the natural frequencies shift. The most sensitive modes are also the most important modes to be estimated for the next parameter estimation.

From Table 2 it can be observed that the high frequency modes are the most sensitive, when considering the magnitude of the frequency shift. A deeper evaluation should also consider the relative frequency shift, corresponding to a $5 \%$ perturbation of the parameter around its hypothetical value (69 GPa) and divided by the respective natural frequency.

\begin{tabular}{rrrrrrrrrrrrrr}
\hline & \multicolumn{10}{c}{ MODES } \\
\cline { 2 - 13 } & $\mathbf{1}$ & $\mathbf{2}$ & $\mathbf{3}$ & $\mathbf{4}$ & $\mathbf{5}$ & $\mathbf{6}$ & $\mathbf{7}$ & $\mathbf{8}$ & $\mathbf{9}$ & $\mathbf{1 0}$ & $\mathbf{1 1}$ & $\mathbf{1 2}$ & $\mathbf{1 3}$ \\
\hline $\boldsymbol{f}[\mathrm{Hz}]$ & 20.4 & 125.3 & 132.5 & 354.1 & 384.9 & 580.6 & 693.6 & 788.8 & 1083.5 & 1135.2 & 1326.4 & 1671.6 & 1677.5 \\
\hline $\boldsymbol{d} \boldsymbol{f}[\mathbf{H z}]$ & 0.5 & 1.5 & 2.5 & 4.3 & 2.8 & 11.4 & 7.1 & 10.7 & 10.8 & 13.7 & 1.7 & 16.7 & 29.0 \\
\hline $\boldsymbol{d} \boldsymbol{f}[\boldsymbol{\%}]$ & 2.3 & 1.2 & 1.9 & 1.2 & 0.7 & 2.0 & 1.0 & 1.4 & 1.0 & 1.2 & 0.1 & 1.0 & 1.7 \\
\hline
\end{tabular}

Table 2: Absolute and relative parameter sensitivities

The last row of Table 2 indicates that all the modes are relatively sensitive to the parameter, except the eleventh mode which is a local mode for the top plate. The first mode is the most sensitive, and the first three modes are chosen for the model order reduction (Figure 3).

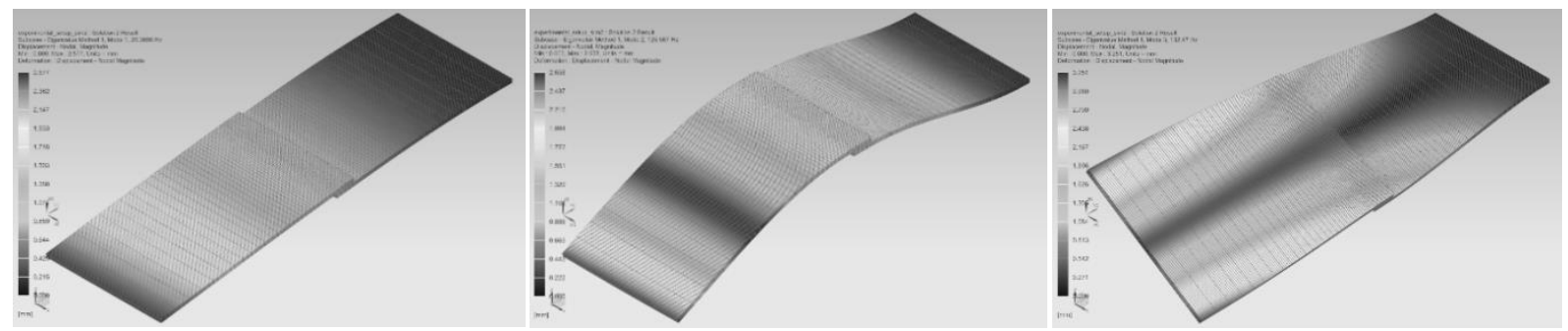

Figure 3: First three modes shapes (selected) 
The model is then reduced from $221760 n_{\text {dofs }}$ to $3 n_{\text {modes }}$, getting computationally lighter and observable with only few measurements as will be discussed in the next section. On the other hand the reduction limits the validity of the model to a frequency band included between $1 \mathrm{~Hz}$ (rigid body displacements excluded) and $150 \mathrm{~Hz}$. These frequency limit is set in between the last included mode $(132 \mathrm{~Hz})$, and the first not included one $(354 \mathrm{~Hz})$, precisely at the frequency value where a reduced model FRF and the corresponding full model FRF amplitudes relatively differ by only the $5 \%$.

The model is experimentally validated from the dynamical point of view: considering an hypothetical value for the parameter to be estimated (69 GPa), its first 3 simulated modal frequencies are well matching with the experimental ones. Particularly the relative error between simulated and experimental modal frequencies stays in a tolerance limit of $\pm 4 \%$. The experimental modal frequencies are retrieved by a simple hammer test elaborated with the Polimax algorithm implemented in Simcenter TestLab 17.

Other parameters have been initially investigated too: the adhesive elastic module and the adhesive length, that could be considered as a damage factor of the joint. However, their sensitivities are far lower, with a maximum $0.05 \%$ of frequency shift relatively to a $5 \%$ change in the parameter value, as can be seen in Figure 4. For this first validation case with the proposed methodology, a higher sensitivity parameter has been chosen, but future efforts towards lowsensitivity estimations will be considered. He [10] [11] and Vaziri [12] already provide experimental sensitivity analysis for a wide variety of parameters in adhesive lap joints, such as thickness, Poisson ratio and void size in the glue layer.

A final remark: from the practical point of view, the need of an accurate identification for low-sensitivity parameters should be properly justified. By definition these parameters have a low influence on the system, and thus an eventual estimation error will also have a low influence. We should anyhow consider that the same parameter could have a weak impact on one modelling domain, for instance the dynamical modelling, but at the same time a strong impact on another modelling domain, for instance the fatigue modelling. It is always recommended to contextualize the parameter to be estimated with the considered modelling domain: in this article all the considerations are related to the structural dynamics aspects.

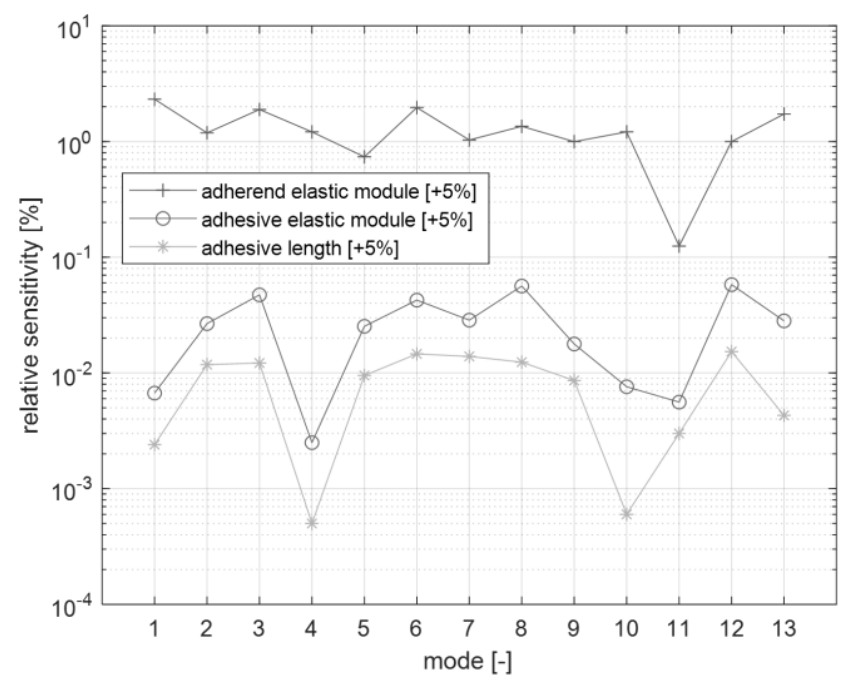

Figure 4: Relative sensitivity of several parameters 


\section{SENSORS CONFIGURATION AND OBSERVABILITY}

The sensors configuration is essential for the proposed methodology, not only because of the observability assessment, but also because the sensors are directly involved in the parameter identification procedure.

A system is observable when, simply put, it is possible to retrieve all its states given a certain set of measurements. For linear systems, as the one considered in this article, there are some well-established observability criteria [2]. The Kalman criterion states that a system is observable if the rank of the observability matrix given by

$$
O B S=\left[\begin{array}{c}
C \\
C A \\
C A^{2} \\
\vdots \\
C A^{n-1}
\end{array}\right]
$$

is equal to $n$, the rank of the dynamical system, i.e. equal to the number of the states. This condition is fundamental for the effectiveness of the linear Kalman filter.

Another method is the Popov-Belevitch-Hautus (PBH) criterion [13], stating that a system is locally observable if the PBH matrix is of full rank for all eigenvalues $\lambda$ of $A$. The PBH matrix is defined as follows:

$$
P B H=\left[\begin{array}{c}
A-\lambda I \\
C
\end{array}\right]
$$

Besides these yes-or-no criteria there are some quantitative assessments to measure the observability, based on cost functions related to the determinant, the condition number or the singular values of the matrixes $O B S$ and $P B H$. Several sensor placement algorithms reported in literature are actually structured on these quantitative assessments [14]. In this case we consider to have only accelerometers available as sensor type. The excitation characteristics are not a priori known, thus the sensor configuration should be generally robust. The method here proposed, aims to maximize the signal to noise ratios. Keeping into account the modal sensitivities with respect to the parameter to be estimated, the mode shapes displacements are at first weighted with the factors given in Table 2 . Then a product-objective function is established (8), and the first sensor is placed at the $(\bar{x}, \bar{y})$ coordinates that maximize the objective function.

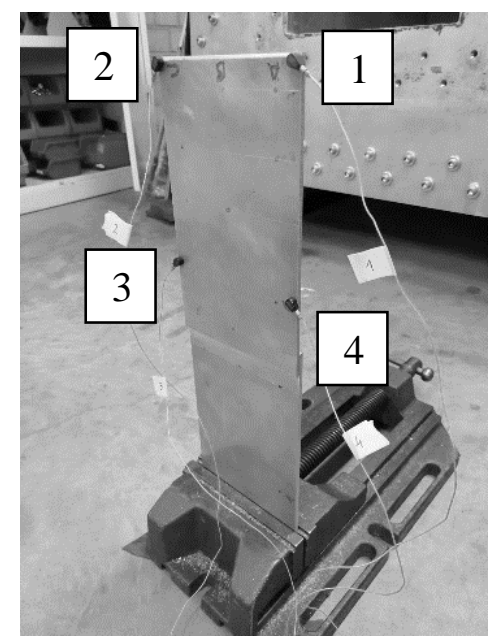

Figure 5: Experimental setup

\begin{tabular}{ccc}
\hline & $\mathbf{X}[\mathbf{m m}]$ & Y $[\mathrm{mm}]$ \\
\hline Accelerometer $\mathbf{1}$ & 0 & 0 \\
\hline Accelerometer $\mathbf{2}$ & 132 & 0 \\
\hline Accelerometer 3 & 132 & 156 \\
\hline Accelerometer 4 & 0 & 156 \\
\hline
\end{tabular}

Table 3: Sensors coordinates resulting from the optimal placement 
In equation (8), $\alpha_{i}$ is the $\mathrm{i}$-th modal weight, proportional to the percentage sensitivity value of Table 2, and $\Psi_{i}(x, y)$ is the i-th displacement mode shape function.

$$
O F_{\text {sensor placement }}=\prod_{i}^{n_{\text {modes }}} \alpha_{i}\left|\Psi_{i}(x, y)\right|
$$

According to this objective function, that considers only the modes of the reduced model, the nodal positions are excluded from the candidate list since they would lead to a zero value for the displacement mode shape function, hence the whole objective function would be zero.

After the first sensor has been selected, the nodes within a certain distance from it are eliminated from the next candidates list, and the procedure continues iteratively, until the defined maximum number of sensors $n_{\text {sens }}$ is reached. As expected from the modes shapes depicted in Figure 3, the 4 accelerometers are located along the edges of the system (Figure 5), precisely at the coordinates reported in Table 3.

Considering the model (4) and measurement equations (5) the observability is positively assessed using the Kalman criteria. In this example, the system is actually observable just with one accelerometer, unless it is located at the clamping. This just means that it is ideally possible to estimate the full states of the system with one only sensor, but it is important to notice that adding more sensor makes the estimation more accurate and it allows to have more information for processing the parameter identification. For these reasons 4 sensors are used.

In order to further extend the methodology to the estimation of local low-sensitive parameters, more complex excitation design and sensor placement strategies should be considered. The location and frequency content of the excitation will play an important role to selectively excite the most sensitive modes.

\section{EXPERIMENTAL DATA}

The methodology proposed in this article is based on a Kalman filter state estimator. Such algorithm requires some measurements and the external disturbances as inputs: in this case the measurements are the 4 acceleration signals and the external disturbance is the force signal given by an hammer hit. Other disturbance types can be used, such as harmonic or random excitations, as long as they adequately excite the modes that are included in the model. The hammer hit is chosen because it requires simple equipment, it is less intrusive than using a shaker or an actuator, and it adequately excite a wide range of frequencies. Future extension of this methodology could include the excitation estimation as output of the Kalman filter, saving the effort of measuring it and possibly allowing the parameter estimation in operational conditions.

The results shown in this work are related to a hit located at the coordinates $\mathrm{x}=20 \mathrm{~mm}$ and $\mathrm{y}=100 \mathrm{~mm}$, hence close to the $4^{\text {th }}$ accelerometer, but other locations have been proved to allow positive estimation too, as long as the states are adequately excited. For instance the mode shapes nodes should be reasonably avoided as excitation location. The data acquisition system used is the portable LMS SCADAS III and the measurement software is Simcenter TestLab17.

Before using the acceleration signals in the estimator, they have been low-pass filtered with a cut-off frequency equal to $150 \mathrm{~Hz}$, corresponding to the validity limit of the reduced model. This operation leads a twofold advantage: some measurement noise is filtered out, and the frequency content of accelerometers is now matching with the frequency content of the reduced order model, both including only the first three modes. In this way both the measurements and the model accuracies are improved. The original accelerometers signals are compared with the low-pass filtered ones in Figure 7. It can be noticed that in the original data all the modes are 
initially excited, also the higher ones that are excluded in the reduced model. For this reason the maximum gap between the measured and the filtered data occurs at the beginning of the time signals, just after the hammer hit. Nevertheless the high modes are quickly damped out and just after one second of measurements, the gap between the measured and filtered signals becomes almost negligible. The excitation signal is time-domain filtered with an exponential window instead, to remove the sensor noise after the impact (Figure 6).

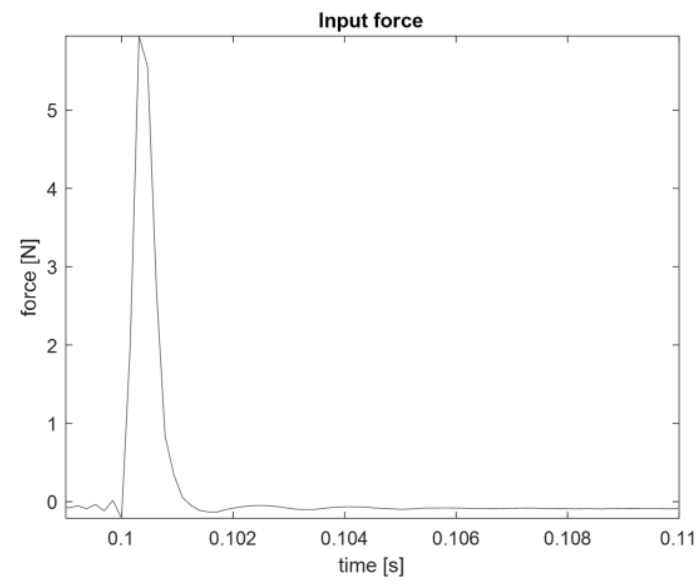

Figure 6: Excitation force signal
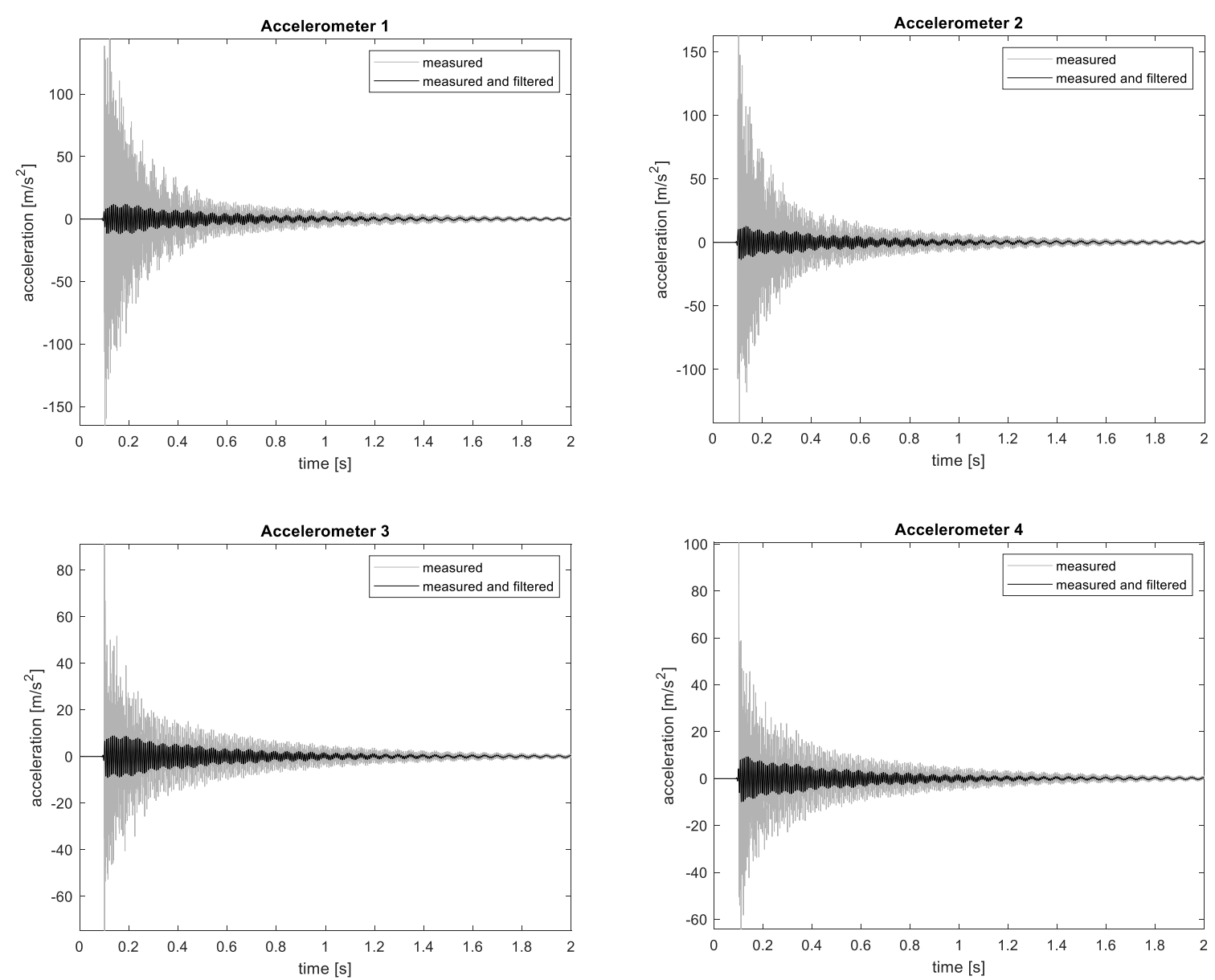

Figure 7: Accelerometers signals 


\section{ESTIMATION}

\subsection{Methodological scheme}

The methodology is structured in a batch of Kalman filter state estimators, each one running for a different value of the parameter to be estimated. It is important to notice that in the current work the parameter is unknown but it is expected to be constant, or at least its dynamics are expected to be orders of magnitude slower than the ones of the states.

Considering that both the measurements and the model are subjected to errors, each Kalman filter estimates 4 virtual accelerometers, that are the virtual representation of the real accelerometers on the test rig. The virtual sensors are expected to give a more accurate and less noisy output w.r.t. to the measurements and the model based forward simulation when separately taken. Then the parameter estimation problem corresponds to a minimization problem with respect to the parameter, where the cost function to be minimized is related to the error between real and virtual sensors.

Specifically, after every run, the error between one real and the relative virtual sensor is integrated over time and normalized with respect to the time integral of the experimental signal. The 4 relative errors, one per sensor, are averaged to obtain the cost function. Then at each parameter value corresponds one Kalman filter run, and further one cost function value. Finally the estimated value for the parameter is the one that minimizes this cost function. The methodology is summarized in the scheme of Figure 8.

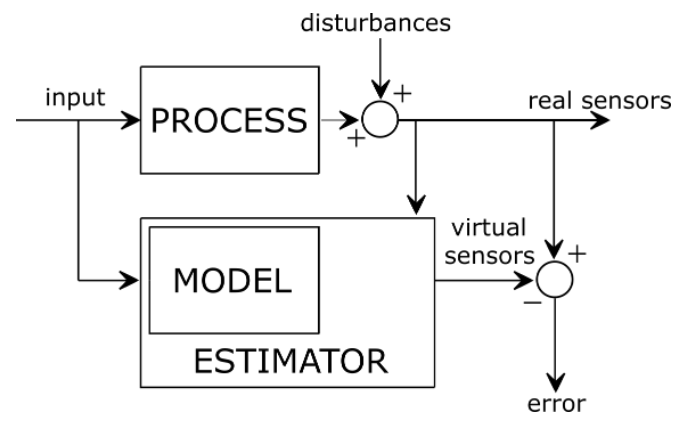

Figure 8: Methodological scheme

$$
C F_{\text {estimation }}=\frac{\int_{0}^{T}\left|y_{\text {real }}(t)-y_{\text {virtual }}(t, p)\right| d t}{\int_{0}^{T}\left|y_{\text {real }}(t)\right| d t}=\frac{\int_{0}^{T}|\operatorname{error}(t, p)| d t}{\int_{0}^{T}\left|y_{\text {real }}(t)\right| d t}
$$

The cost function is described in equation (9), where $y_{\text {real }}$ is the vector of time-domain signal extracted from the real sensors, $y_{\text {virtual }}(t, p)$ is the vector of time-domain estimated signal, i.e. the virtual sensors signal. Summarizing the methodology proposes to identify system parameters by fitting the estimation to the measurement, rather then directly the forward simulation to the measurement. The estimation is more accurate and better synchronized to the measurements, and this allows the time-domain fitting.

\subsection{Kalman filter}

A Kalman filter is a stochastic estimator that can be used to estimate states, input and parameters, combining together a mathematical model and experimental measurements [5]. In this case the model is assumed to be linear, since only small displacements are expected, and there are not any strong geometric nor material nonlinearities. At each time-step, an a priori 
estimation is elaborated based solely on the model; secondly the virtual measurements are computed based on the a priori estimation, the error between them and the real current measurements is calculated and weighted considering the known model and measurements uncertainties; with this information the a priori estimation is finally corrected with the a posteriori one. Before proceeding to the next time-step the relative process-measurements uncertainty is updated, taking into account the covariance of the estimation.

Before starting the Kalman filter time loop, the model is time-discretized with exponential integration, that leads to:

$$
\begin{gathered}
F=e^{A \Delta t} \\
G=F\left[I-e^{-A \Delta t}\right] A^{-1} B \\
x_{k}=F x_{k-1}+G u_{k-1} \\
y_{k}=C x_{k}+D u_{k}
\end{gathered}
$$

After the time discretization, the Kalman filter procedures described above are applied timestep by time-step:

$$
\begin{gathered}
P_{k}^{-}=F P_{k-1}^{+} F^{T}+Q \\
K_{k}=P_{k}^{-} C^{T}\left(C P_{k}^{-} C^{T}+R\right)^{-1} \\
\hat{x}_{k}^{-}=F \hat{x}_{k-1}^{+}+G u_{k-1} \\
\hat{x}_{k}^{+}=\hat{x}_{k}^{-}+K_{k}\left[y_{k}-\left(C \hat{x}_{k}^{-}+D u_{k}\right)\right] \\
P_{k}^{+}=\left(I-K_{k} C\right) P_{k}^{-}\left(I-K_{k} C\right)^{T}+K_{k} R K_{k}^{T}
\end{gathered}
$$

In these mathematical formulations $\hat{x}_{k}^{-}$is the a priori estimation vector related to the $\mathrm{k}$-th time-step, $\hat{x}_{k}^{+}$is the a posteriori one, $P_{k}^{-}$is the a priori covariance matrix, $P_{k}^{+}$is the a posteriori covariance matrix, $Q$ is the process/model covariance matrix and $R$ is the measurements covariance matrix. Finally $K_{k}$ is the Kalman gain, that weights the relative reliability of the measurements with respect to the model.

\subsection{Model and measurements uncertainties}

The Kalman filter is defined stochastic estimator because it takes into account the expected uncertainties deriving from the measurements and the model. Measurements uncertainty is clearly due to the sensors noise, sensor nonlinearities, but also to eventual systematic errors such as imperfect calibration, fastening, external influences et cetera. On the other side, a model is by definition an approximation of the reality and as such, it always brings an error with itself. Some of the possible causes of this error are the neglected un-modelled behaviors, the parameters setting, the time and space discretization and last but not least the boundary conditions definition.

Within the Kalman filter algorithm the errors distribution is approximated to be Gaussian with mean equal to zero. The errors are then exclusively defined by their covariance values, indicated in the $n_{\text {sens }} \times n_{\text {sens }}$ diagonal matrix $R$ for the measurements, and in the $2 n_{\text {modes }} \times 2 n_{\text {modes }}$ diagonal matrix $Q$ for the states. The covariance setting is a crucial step for the estimation results, particularly in terms of relative weight between $R$ and $Q$. As mentioned above, this ratio will lead the filter to adjust the Kalman gain on every time-step.

If for the measurements it is reasonable to link their covariance to the sensor characteristics available on their data-sheets, it is less straightforward to set the model covariance, especially when the states are the modal contributions. In this work the derivatives covariance is set to zero. The non-derivatives standard deviation is initially set to a value two orders of magnitude 
lower than the forward-simulated modal contributions (19).

$$
Q=\left[\begin{array}{cccccc}
10^{-16} & 0 & 0 & 0 & 0 & 0 \\
0 & 10^{-16} & 0 & 0 & 0 & 0 \\
0 & 0 & 10^{-16} & 0 & 0 & 0 \\
0 & 0 & 0 & 0 & 0 & 0 \\
0 & 0 & 0 & 0 & 0 & 0 \\
0 & 0 & 0 & 0 & 0 & 0
\end{array}\right]
$$

Setting $Q$ as a diagonal matrix does not consider the eventual cross-coupled states errors. In order to extend the methodology to this case, the continuous-time $Q$ is integrated with the procedure developed by Van Loan [15], and the discrete-time $Q_{D}$ is obtained as a full matrix and used in the Kalman filter instead of $Q$.

\subsection{Results}

The expected value for the aluminum elastic module is $69 \mathrm{GPa}$. Figure 9 and Figure 10 are showing the results of the Kalman filter when the parameter value is fixed at the expected one. At first, in Figure 9 the plot emphasizes the added value of using a closed-loop estimator rather than a forward simulation. In terms of amplitudes of the full time signal, the forward-simulation seems matching quite well the measurements, but when zooming in it is possible to quantify a significant gap between amplitudes and phases. The estimated acceleration is almost perfectly matching the measured one, instead.
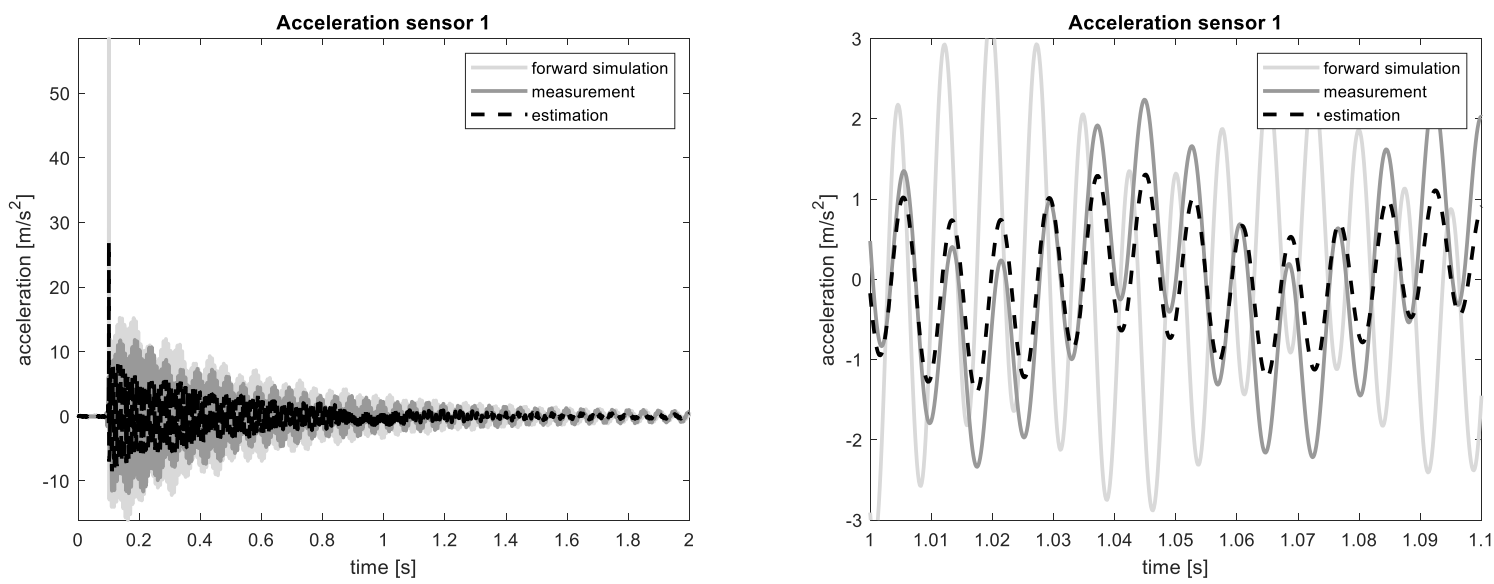

Figure 9: Full-time signal at accelerometer 1 (left), zoomed signal at accelerometer 1 (right)

In Figure 10 the forward-simulation is not represented anymore, to give more visibility to the comparison between measurements and estimations.
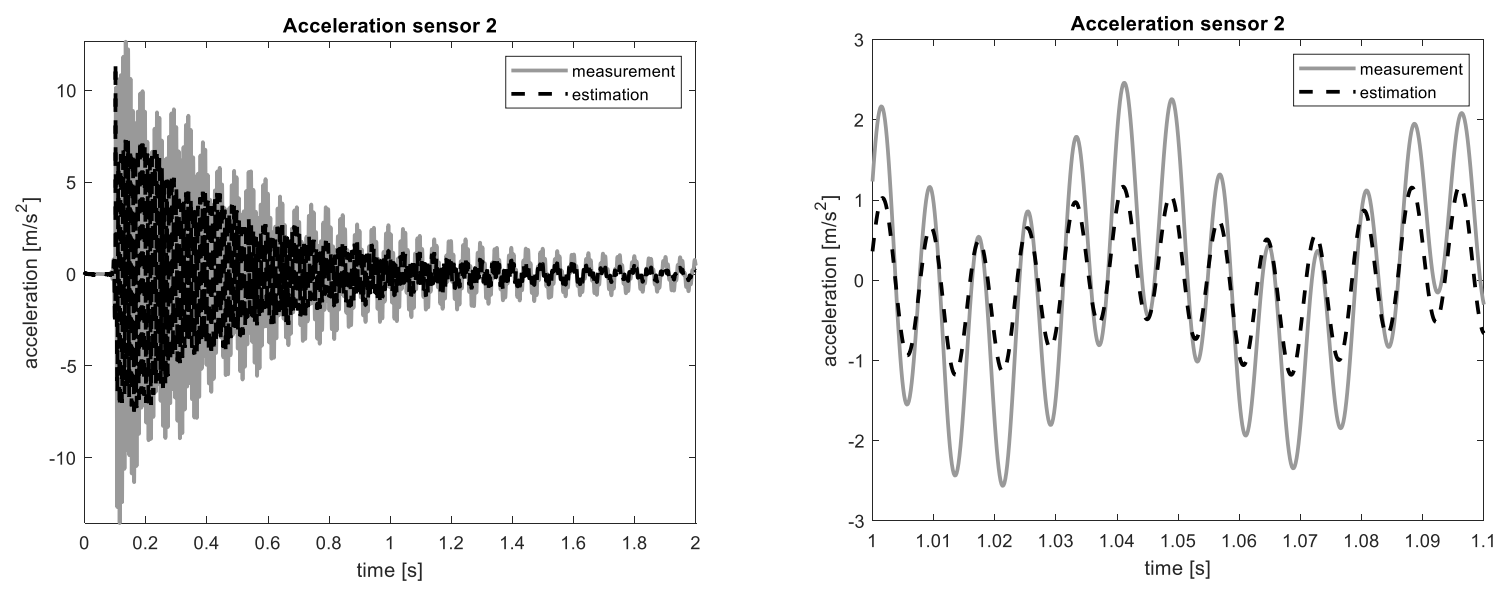

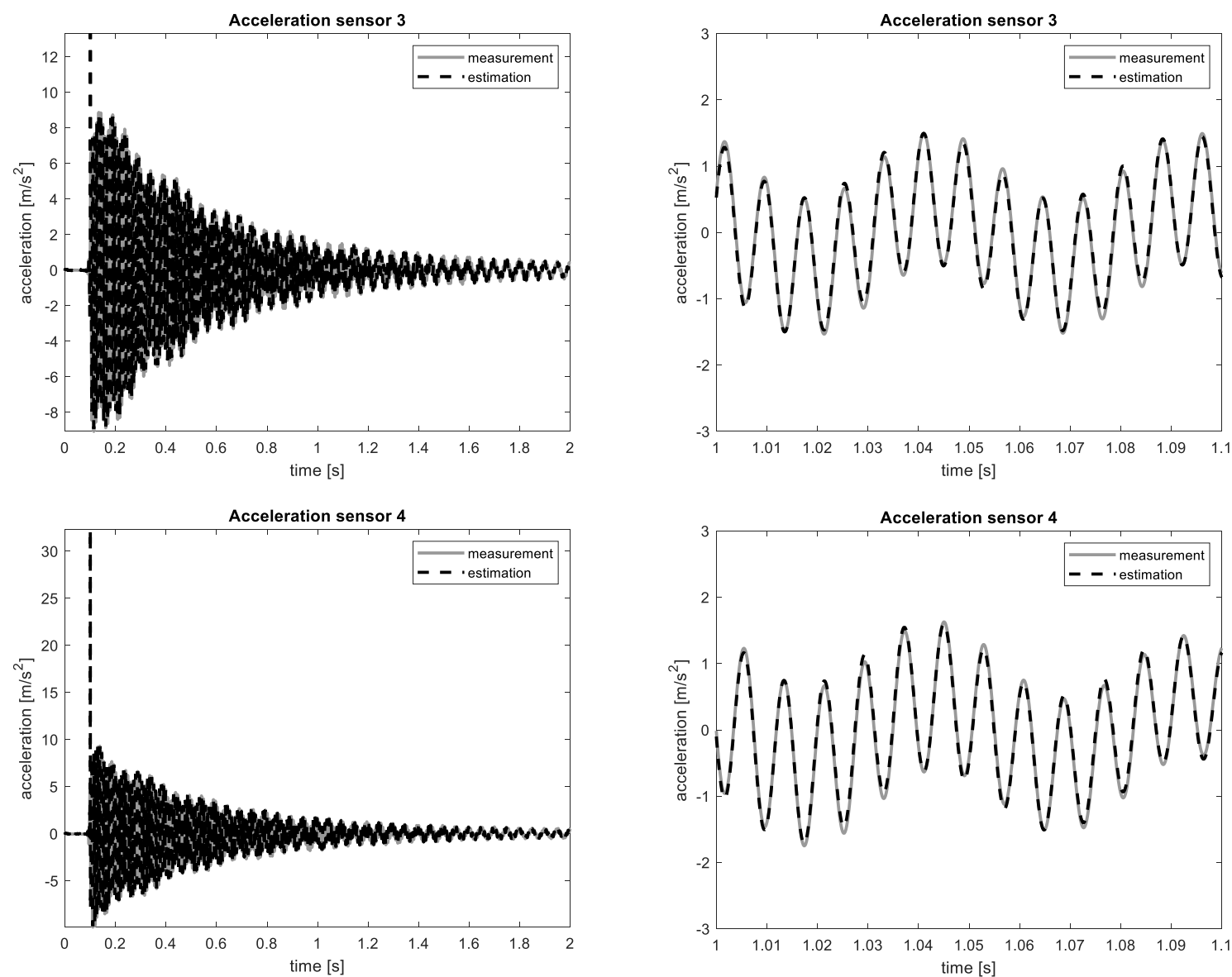

Figure 10: Full-time signal at accelerometers 2,3,4 (left), zoomed signal at accelerometers 2,3,4 (right)

The estimation at the accelerometers 3 and 4 seem to be more accurate, actually it is almost perfectly matching the measurements, probably because they are closer to the excitation source, then the model transfer path is shorter and less influent. Further investigation will be carried on this observation. A similar observation will hold for Figure 11 where the accelerometer 3 and 4 provide a more evident estimation convergence.

As mentioned earlier the Kalman filter is repeated for different values of the parameter, and for each run the cost function described in equation (9) is recorded.
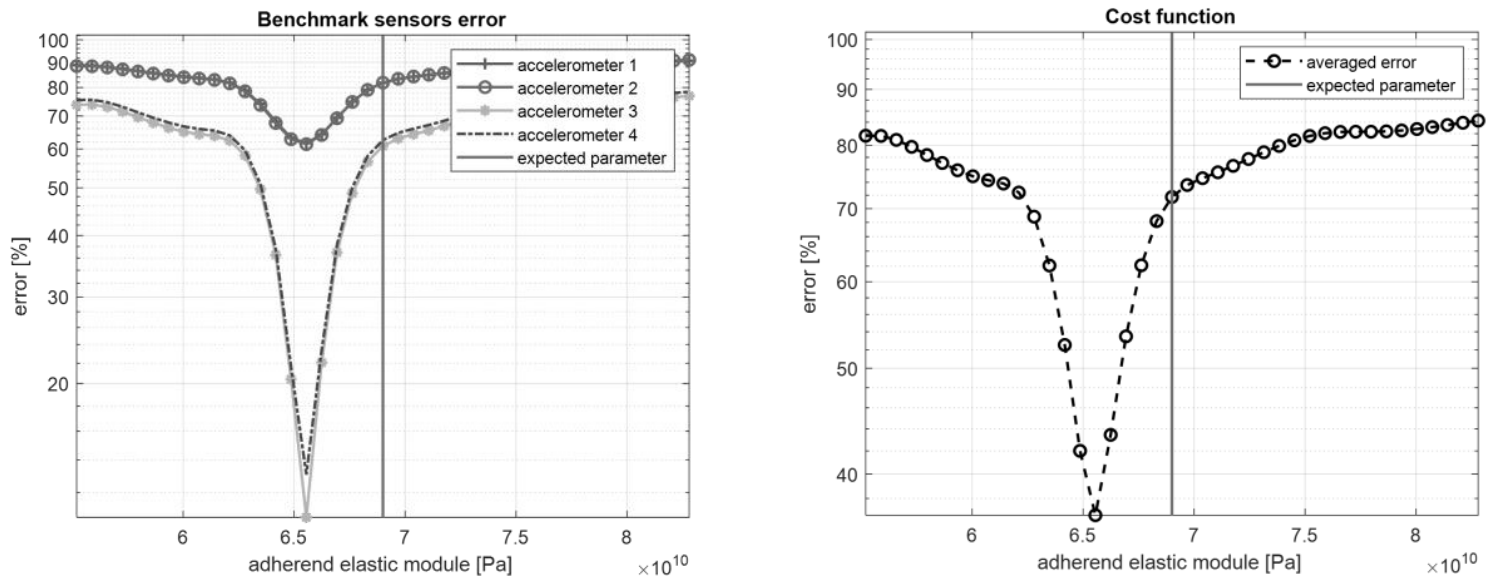

Figure 11: Benchmark sensors errors (left), and cost function (right), for different values of adherend elastic module

These recorded values are reported in Figure 11, where the cost function in the right plot is 
representing the average between the 4 curves in the left plot. It can be observed that the parameter estimation converges to the value of $66 \mathrm{GPa}$, slightly lower than the expected $69 \mathrm{GPa}$. This offset occurs because the clamping is modelled as ideally stiff, whereas in reality it always allows some flexibility. In this exercise, since the adherend (i.e. the clamped plate) elastic module is the only tunable parameter for a good match between experimental and model system flexibility, its estimation takes also into account the clamping flexibility, resulting in a lower than expected value. The estimated value, apparently off, anyway allows the updated model to provide results that are closer to the reality, thus more accurate. As a future step a multiparameter estimation will be considered, in which the clamping stiffness will be included in the model and estimated alongside the elastic module of the clamped plate.

Taken the 4 accelerometers errors separately, they still converge to the same parameter, meaning that in future even less intrusive setups will be possible. Nevertheless it is important to notice that the accelerometer positions have an influence on the clearness of the convergence. As already observed before in Figure 9 and Figure 10, the sensors number 3 and 4 show a lower error with respect to the others, resulting in a more evident parameter estimation (Figure 11). Another future improvement could involve the resolution of the parameter estimation: the parameter space could be further finer sampled in the surroundings of the first estimation and the same minimization procedure could be repeated until the desired precision.

Finally a consideration about the influence of the model uncertainty, i.e. the $Q$ matrix. As mentioned before, its setting has usually a big impact on the states estimation, and then also on the proposed parameter estimation methodology. For this final investigation, other values for the model standard deviations (square root of the $Q$ matrix diagonal) have been considered, linearly sampled around the initial value $10^{-8}$, within a range that begins from $10^{-20}$ and ends at $10^{\circ}$. Once more, the cost function has been recorded for each combination of parameters and model standard deviation values, and the results are represented in the 3D plot of Figure 12.

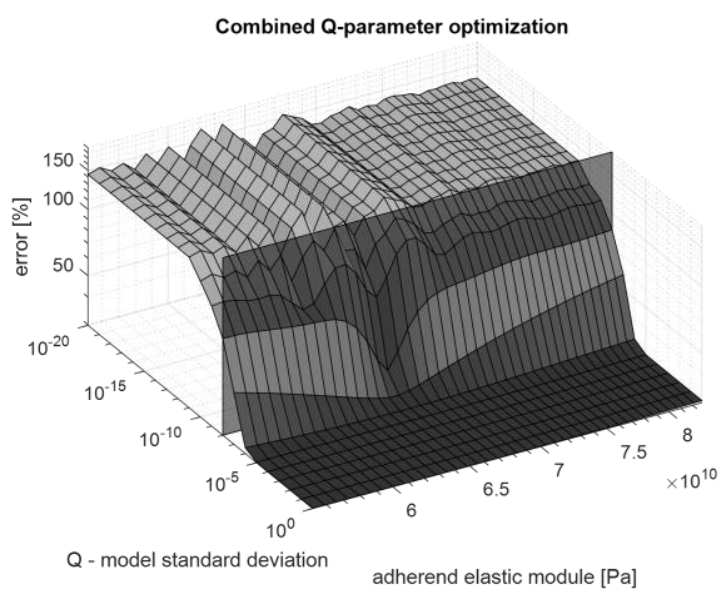

Figure 12: Influence of the model uncertainty on the parameter estimation

High values of $Q$ mean that the model uncertainty is high, then the Kalman filter gives high confidence to the measurements and the virtual sensors signals are perfectly matching the real ones. As a direct consequence, the error tends to a minimum when $Q$ tends to its maximum plotted value (Figure 12). On the other hand, low values of $Q$ mean that the model is almost perfect, then the algorithm ignores the measurements, and the estimation is nothing else than a forward-simulation. In this case the error is always high and the parameter estimation is not possible. In between these two sectors, the Figure 12 shows an intermediate region, where the influence of the parameter on the error is more evident. Then in conclusion, the model standard 
deviation must be set to a value within this area to make the estimation possible. The transparent vertical plane shows that the initial setting of the model standard deviation, equal to $10^{-8}$ indeed enables the estimation. The intersection curve between the vertical plane and the 3D surface exactly corresponds to the right plot in Figure 12.

\section{CONCLUSIONS}

Within the modelling of lightweight assemblies, where different types of material are connected together with several structural joints, the parameters identification is challenging. Often these parameters are local and it is not easy to access them, often they do not even have a direct physical link. In this context, this work introduces and experimentally validates a novel virtual sensing technique for parameter estimation. The methodology is non-intrusive, as it does not require the sensors and the excitation sources to be located directly on the component which the parameter belongs to. The methodology can be applied in-situ, i.e. keeping the system in its operating conditions, without dismounting the component which the parameter belongs to. The estimation of the elastic module of a plate in a single lap adhesive joint has been chosen as experimental validation case. The technique consists in the minimization, with respect to the parameter, of the error between the real sensors placed on the test rig, and the virtual ones estimated through a Kalman filter. The same approach can be applied not only for joint substrates estimation, but also for the identification of the connecting elements themselves, such as adhesive layers, bolts, rivets.

The article proposes a modelling strategy for the adhesive connection, a parametric model order reduction, and it suggests how to properly select the reduced model modes according to the sensitivity and observability assessments. Some considerations about the Kalman filter configuration can be extended for other state/parameter/input estimation exercises in which the states are the modal contributions: the frequency content of the measurements should be adjusted to match the model one, the model covariance has a big impact on the estimation result and this work provides some guidelines on its setting. Another basic but significant observation about identification problems is that all the approximation errors of the model are condensed in the estimated parameter. Thus it should not be considered as the real parameter, but rather the parameter that allows the model to better match the reality.

Finally this work opens the way to some possible next steps. Multi-parameter estimations could limit the condensation of the approximation errors in one only parameter. In order to make this extension possible, different sampling techniques for the parameter space should be considered. Another challenge mentioned in this paper is the estimation of low-sensitivity parameters. If this is required, more complex and tailored strategies should be taken into account for the sensors placement and the excitation design.

\section{ACKNOWLEDGEMENTS}

This research was partially supported by Flanders Make, the strategic research center for the manufacturing industry. 


\section{REFERENCES}

[1] M. R. W. Brake, "An overview of the analysis of jointed structures," in The mechanics of jointed structures, Houston, Springer, 2018, pp. 11-24.

[2] J. Croes, Virtual sensing in mechatronic systems, Leuven: Faculty of Engineering Science, 2017.

[3] J. E. Mottershead and M. I. Friswell, "Model updating in structural dynamics: a survey," Journal of Sound and Vibration, pp. 347-375, 1993.

[4] K. J. Åström and P. Eykhoff, "System identification - A survey," Automatica, pp. 123$162,1971$.

[5] D. Simon, Optimal state estimation, Hoboken: John Wiley \& Sons, Inc., 2006.

[6] X. He, "A review of finite element analysis of adhesively bonded joints," International Journal of Adhesion and Adhesives, pp. 248-264, 2011.

[7] L. Van Belle, D. Brandolisio, E. Deckers, S. Jonckheere, C. Claeys, B. Pluymers and W. Desmet, "Experimental validation of numerical structural dynamic models for metal plate joining techniques," Journal of Vibration and Control, pp. 1-22, 2017.

[8] P. Benner, S. Gugercin and K. Willcox, A survey of model reduction methods for parametric systems, Max Planck Institute Magdeburg Preprint MPIMD/13-14, 2013.

[9] F. Naets, J. Croes and W. Desmet, "An online coupled state/input/parameter estimation approach for structural dynamics," Comput. Methods Appl. Mech. Engrg, 2014.

[10] X. He and S. O. Oyadiji, "Influence of adhesive characteristics on the transverse free vibration of single lap-jointed cantilevered beams," Journal of Materials Processing Technology, 2001.

[11] X. He, "Numerical and experimental investigations of the dynamic response of bonded beams with a single-lap joint," International Journal of Adhesione \& Adhesives, pp. 79$85,2012$.

[12] A. Vaziri, H. Nayeb-Hashemi and R. Hamidzadeh H, "Experimental and analytical investigations of the dynamic response of adhesively bonded single lap joints," Journal of Vibration and Acoustic, 2004.

[13] S. Ghosh and J. Rosenthal, "A generalized Popov-Belevitch-Hautus test of observability," IEEE Transactions on Automatic Control, vol. 40, pp. 176-180, 1995.

[14] T. Tamarozzi, E. Risaliti, W. Rottiers, K. Janssens and W. Desmet, "Noise, illconditioning and sensor placement analysis for force estimation through virtual sensing".

[15] G. H. Golub and C. F. Van Loan, "Matrix computations," JHU Press, 2012, p. 246. 